\title{
Les données probantes sont-elles si importantes?
}

\author{
par Kevin W Hall
}

$\grave{A}$

la mi-quarantaine, j’ai commencé à jouer au golf pour vite

me rendre compte que c'était un sport difficile. Après plus de dix ans, ainsi que de nombreuses parties et leçons de golf, j'estime toujours que je connais une excellente journée si je joue moins de 100 sur un parcours de 18 trous. Ma seule consolation, c'est que je ne suis pas seul : en effet, selon quelques livres que j’ai lus sur le sujet, moins de $10 \%$ des golfeurs obtiendront bien légitimement un score inférieur à 100. J'étais donc un peu sceptique lorsque, après la mort récente de Kim Jong Il, le "Grand Leader " de la Corée du Nord, j’ai lu les exploits miraculeux qu'on lui a reconnus, y compris qu’à sa toute première partie de golf, il aurait réussi près de douze trous en un consécutifs ${ }^{1}$.

Nombre de ses autres exploits miraculeux semblent tout aussi invraisemblables. En fait, beaucoup d'entre vous se demandent peut-être, tout comme je me le suis demandé, si quelqu'un en Corée du Nord prend vraiment ces prétentions au sérieux. Dans un article paru dans le Globe and Mail ${ }^{1}$ peu après Noël 2011, on rapportait que l'observateur canadien en Corée du Nord et ancien résident de ce pays, Erich Weingartner, avait dit que « Beaucoup de gens considèrent encore Kim Il-sung [le premier des trois dirigeants de la famille Kim et père de Jong-il] comme un véritable dieu." Lorsqu'on lui a demandé son opinion, le professeur Paul Evans, directeur de l'Institute of Asian Research de l'Université de Colombie-Britannique, nous a mis en garde de ne pas conclure que les Nord-Coréens sont plus enclins à croire à l'improbable que nous le sommes. Après tout, soulignait-il, les Nord-Américains viennent tout juste de célébrer la fête de Noël, alors que les chrétiens croient véritablement en l'enfant né de la vierge Marie, aux anges descendus du Ciel et à une étoile guidant les mages dans la nuit vers leur destination.

Mais qu'est-ce que tout cela a à voir avec la pharmacie? Eh bien, pour commencer, la foi est un trait extrêmement important de l'humain. On ne peut pas tout prouver ou réfuter. Nous pouvons croire que quelqu'un nous aime, mais la science peut-elle vraiment le prouver ou le réfuter? Devrions-nous ne plus croire à l'amour sur la base que la science ne peut le prouver? En revanche, lorsqu'une question peut être soumise à

la recherche scientifique, quelle obligation avons-nous, en tant que professionnels, lorsque les croyances et la science se heurtent? La pharmacie fait face à nombre de questions pour lesquelles il y a de bonnes raisons de croire que nous, en tant que professionnels, omettons de tenir compte des contradictions entre les données disponibles et nos propres réactions au regard de ces données.

La médecine douce est l'une de ces zones grises. Dans son livre Snake Oil Medicine: The Truth About Complementary and Alternative Medicine ${ }^{2}$, Bausell argumente qu'à peu d'exceptions près, la plupart des traitements de la médecine douce n'exercent aucun effet intrinsèque bénéfique. Cela ne veut pas dire que les patients n'éprouvent pas ce qu'ils perçoivent comme étant des effets bénéfiques, mais plutôt que les effets bénéfiques peuvent être en grande partie, sinon complètement, attribuables à toute une gamme d'effets psychologiques et physiologiques qui, collectivement, constituent ce que nous connaissons sous le nom d'effet placebo. Malgré les données probantes en ce sens qui se sont accumulées ces dernières années, Bausell reconnaît lui-même que si les tendances actuelles se poursuivent, la plupart des Nord-Américains, du moins ceux qui peuvent se le permettre, auront recours aux pratiques de la médecine douce. Reconnaissant cette réalité non fondée sur des données probantes, beaucoup de pharmacies communautaires élargissent leurs offres de produits de médecine douce, les facultés de pharmacie commencent à offrir des cours dans ce domaine et des établissements d'enseignement, publics et privés, proposent des programmes de certificats dans divers domaines de pratique de la médecine douce. En fait, la médecine douce a atteint un renom tel que l'on qualifie d'étroitesse d'esprit le fait de s'interroger sur les mérites de ses traitements même si, pourtant, ils sont rarement étayés du type de données scientifiques probantes nécessaires à la médecine traditionnelle. Certains diront que la médecine douce est au pire sans danger, mais ce n'est pas toujours le cas. Des effets secondaires graves surviennent vraiment ${ }^{3,4}$. De plus, est-il approprié d'ignorer le fait que d'imposantes sommes d'argent sont dépensées pour des produits quasiment ou complètement dépourvus de données prouvant leurs prétendus bienfaits? 
En regardant les données d'un autre point de vue, nous pouvons nous demander comment les praticiens en pharmacie réagissent lorsqu'il y a des données raisonnablement robustes pour éclairer nos décisions. Les trois derniers rapports sur les pharmacies hospitalières canadiennes au Canada avaient tous une section qui examinait le lien entre la façon dont les services de pharmacie priorisaient les diverses activités de la pharmacie et les données à l'appui de la valeur de ces activités. Ces résultats suggèrent un écart significatif entre les données probantes et la priorisation des activités de la pharmacies.

Un autre exemple qui illustre le triste retard sur les données probantes est celui de l'adoption des nouveaux rôles professionnels dont il a été montré qu'ils amélioraient les résultats thérapeutiques ${ }^{6-9}$. Les Canadiens ne tirent pas tous les avantages des soins d'un pharmacien, à cause de la lenteur et de l'apparente réticence de la part de la profession à aller de l'avant avec l'élargissement du champ de pratique, dont les preuves scientifiques montrent pourtant clairement le bien-fondé.

Dans leur livre de 2009 intitulé Unscientific America: How Scientific Illiteracy Threatens our Future ${ }^{10}$, Mooney et Kirshenbaum ont sonné l'alarme concernant le fait qu'on ignore de plus en plus les preuves scientifiques dans notre société moderne. Peut-être devrions-nous nous demander si, en toute honnêteté, les données probantes servent réellement à quelque chose dans la pratique de la pharmacie. Utilisons-nous vraiment les données probantes pour décider de ce que nous devrions faire ou ne pas faire comme profession? Sur une note plus personnelle, chacun de nous peut se poser les mêmes questions : Est-ce que j'exerce mes activités au quotidien en me fondant sur des données probantes ou sur mes préférences? Suis-je prêt ou prête à centrer ma pratique sur des activités dont il a été montré qu'elles amélioraient les résultats thérapeutiques, comme le bilan comparatif des médicaments? Est-ce que j’appuie la mise en œuvre d'attentes et de responsabilités inhérentes à la pratique fondée sur des données probantes ou est-ce que je rejette cette idée sur la base qu'il s'agit d'une autre approche «à l'emporte-pièce » de la pratique de la pharmacie?

Pour terminer, j'ai un aveu à faire : je m'attends toujours à frapper un coup de départ au Tournoi de golf des maîtres d'ici peu, malgré toutes les données probantes qui s'accumulent en ma défaveur depuis les 10 dernières années!

[Traduction par l'éditeur]

\section{Références}

1. Reeder M. Sifting through the golf sands for a hint of North Korea's future. Globe and Mail [Toronto, ON]: 30 décembre 2011. Publié au http:// vtncankor.wordpress.com/2012/01/05/sifting-through-the-golf-sands-fora-hint-of-north-koreas-future/. Consulté le 28 février 2012.

2. Bausell RB. Snake oil science: the truth about complementary and alternative medicine. New York (NY): Oxford University Press; 2007.

3. Niggemann B, Grüber C. Side-effects of complementary and alternative medicine. Allergy 2003;58(8):707-716.

4. Ernst E. Heavy metals in traditional Indian remedies. Eur J Clin Pharmacol 2002;57(12):891-896.

5. Bussières JF. Services cliniques de pharmacie. Dans : Babich M, Bornstein C, Bussières JF, Hall K, Harding J, Lefebvre P, et al., rédacteurs. Rapport annuel 2009-2010 sur les pharmacies hospitalières canadiennes. Eli Lilly; 2010. p. 3-22. Publié au www.lillyhospitalsurvey.ca/hpc2/content/ 2010_report/chapter_bF\%20.pdf. Consulté le 28 février 2012.

6. Tsuyuki RT, Johnson JA, Teo KK, Simpson SH, Ackman ML, Biggs RS, et al. A randomized trial of the effect of community pharmacist intervention on cholesterol risk management: the Study of Cardiovascular Risk Intervention by Pharmacists (SCRIP). Arch Intern Med 2002; 162(10):1149-1155.

7. McLean DL, McAlister FA, Johnson JA, King KM, Makowsky MJ, Jones $\mathrm{CA}$, et al. A randomized trial of the effect of community pharmacist and nurse care on improving blood pressure management in patients with diabetes mellitus: study of cardiovascular risk intervention by pharmacists-hypertension (SCRIP-HTN). Arch Intern Med 2008; 168(21):2355-2361.

8. Chabot I, Moisan J, Grégoire JP, Milot A. Pharmacist intervention program for control of hypertension. Ann Pharmacother 2003;37(9): 1186-1193.

9. Koshman SL, Charrois TL, Simpson SH, McAlister FA, Tsuyuki RT. Pharmacist care of patients with heart failure: a systematic review of randomized trials. Arch Intern Med 2008;168(7):687-694.

10. Mooney C, Kirshenbaum S. Unscientific America: how scientific illiteracy threatens our future. New York (NY): Basic Books, A Member of the Perseus Books Group; 2009.

Kevin W Hall, B. Sc. Pharm., Pharm. D., FCSHP, est professeur agrégé de clinique à la Faculté des sciences pharmaceutiques, University of Alberta, Edmonton, Alberta. Il est également rédacteur adjoint du JCPH.

\section{Adresse de correspondance :}

Dr Kevin W Hall

Faculty of Pharmacy and Pharmaceutical Sciences

University of Alberta

ECHA 3-228

Edmonton $A B$ T6G 1C9

Courriel : khall@pharmacy.ualberta.ca 